\title{
An equation of state for porous materials under shock loading
}

\author{
N RAMAKRISHNAN and K SIVAKUMAR \\ Defence Metallurgical Research Laboratory, Hyderabad 500 258, India \\ MS received 3 November 1987; revised 30 January 1988
}

\begin{abstract}
Based on the physical interpretation of the linear equation of state (EOS) of dense solids under shock loading, which relates particle and shock speeds as $U_{s}=C_{b}+g U_{p}$, the EOS for porous solids has been developed and is expressed as $U_{s}^{*}=\Psi C_{b}^{*}+g^{*} U_{p}$ where $C_{b}^{*}$ and $g^{*}$ are effective bulk sound speed and effective inverse ultimate volume strain respectively. $\Psi$ is a pore collapse function introduced specially to differentiate loading and unloading behaviour. $C_{b}^{*}$ and $g^{*}$ are derived theoretically whereas $\Psi$ is established empirically as $\Psi=f\left(U_{p}, C_{b}\right)$. This EOS does not call for any experimentally established material constant to describe the effect of porosity. Also its ability to describe the unloading behaviour distinguishes it from the presently available equations of state.
\end{abstract}

Keywords. Shock loading: porous materials; equation of state.

\section{Introduction}

The study of shock wave propagation in porous materials has several applications. One of the important applications is shock consolidation of metallic and ceramic powders. Apart from this, the study is useful in understanding the behaviour of materials under shock loading at high temperatures and internal energy levels, as porous samples are used in the experiments conducted for this purpose. One of the applications in defence is the development of porous armour materials to absorb the shock induced by explosion or high velocity penetration. The primary requirement of all these applications is to understand the physics of the material response to shock loading.

Essentially, a porous material, subjected to shock loading is densified almost instantaneously. At low pressures of shock loading, pore collapse takes place by shearing of the pore walls and at relatively high pressures this may even lead to localized melting. In order to quantify this behaviour, the basic Hugoniot equations of dense solid developed based on conservation of mass, momentum and energy have been directly adapted. Nevertheless, these equations cannot completely describe the behaviour without an equation of state (EOS). Though a few equations of state are presently available, most of them call for experimentally established material constants corresponding to porous materials. Moreover, the P-V Hugoniots of loading and release of shock waves do not coincide in the case of porous solids unlike those of dense solids and the quantitative understanding of release waves is very little. Therefore, an attempt was made to develop a suitable EOS based on the linear relationship between particle speed and shock speed. A special pore collapse function has been introduced which modulates the bulk sound speed of the porous solid in order to describe both loading and unloading behaviour. 
The earliest investigation in this subject was by Al'tshuler et al (1958) wherein EOS was established semi-empirically. Herrmann (1969) suggested an elegant exponential equation popularly known as $p-\alpha$ model for fitting the experimental data. Butcher (1971) suggested a rate-dependent model which considers even elastic compression and hence particularly suitable for low pressure regions. But this analysis involves trial values for the constants, numerical solution of the problem, comparison with experimental data and finally readjustment of the constants to make the solutions agree with the experimental data for each material. Roman and Gorobtsov (1981) adapted the linear EOS and in this analysis, the compaction ratio of a porous solid as a whole is determined by compression ratio of the pore and the material. Basically, the rule of mixtures has been assumed in establishing the effective compaction ratio. Theoretically, the rule of mixtures corresponds to only the upper limit (Christensen 1979).

McQueen et al (1970) used the equations relating the change in internal energy and the change in pressure through Grüneisen parameter, in which volume dependency of Grüneisen parameter is established based on maximum volume strain possible corresponding to infinite pressure. In this case, shock speed calculated as a function of particle speed fits very well with the experimental data, but no specific unloading Hugoniot has been suggested. Lotrich et al (1986) derived a simple equation using phenomenological approach assuming every particle in a powder agglomerate to be moving freely in the pore space and striking another particle and thus propagating the shock. This model yields simple relation between particle and shock speeds but does not explain the phenomenon of unloading.

Derek Raybould (1981) constructed the EOS based on quasi-static pressuredensity relationship. Linde and Schmidt (1966), Zel'dovich and Raizer (1967) and Simons and Legner (1982) proposed similar equations in which the Grüneisen parameter of the porous material is established experimentally.

\section{Physical basis}

The Rankine-Hugoniot equations for dense continuum are given as

$$
\begin{aligned}
& U_{s}=\rho_{0}\left(U_{s}-U_{p}\right), \\
& P-P_{0}=\rho_{0} U_{s} U_{p}, \\
& {\left[\left(E-E_{0}\right)+U_{p}^{2} / 2\right] \rho_{0} U_{s}=P U_{p},}
\end{aligned}
$$

where $U_{p}$ is the particle velocity, $U_{s}$ the shock velocity, $\rho$ the density, $P$ the pressure and $E$ the internal energy (subscript zero corresponds to initial conditions). These equations are based on the conservation of mass, momentum and energy respectively. If these equations have to be directly adapted to porous materials, an EOS has to be developed with which porous material could be treated as a continuum. One of the means' to achieve this is to modify the EOS corresponding to the dense state of the porous material appropriately, by implicitly incorporating the effect of porosity, assuming the pore distribution to be statistically homogeneous.

In the case of dense material, one of the forms used to represent the EOS is the linear relationship between particle speed and shock speed which is given as

$$
U_{s}=C_{b}+g U_{p},
$$


where $C_{b}$ and $g$ are the experimentally established material constants. In the majority of the cases, $C_{b}$ corresponds to the bulk sound speed and $g$ to an ultimate state of compression where a volume strain of $1 / \mathrm{g}$ corresponds to a state of infinite pressure.

For establishing these constants for porous solids, $C_{b}$ and $g$ of the dense material have to be modified to take porosity into account and in this paper they are denoted as $C_{b}^{*}$ and $g^{*}$. Apart from this, the bulk sound speed itself is modulated by the collapse of pores and the modulating function is denoted as $\Psi$. Thus, the EOS for a porous material is written in the form

$$
U_{s}^{*}=\Psi C_{b}^{*}+g^{*} U_{p},
$$

where $U_{s}^{*}$ is the speed of shock wave in the porous solid.

\section{Evaluation of effective bulk sound speed $C_{b}^{*}$}

The bulk sound speed $C_{b}$ is related to the bulk modulus $K$ and the material density $\rho$, in a dense solid as

$$
C_{b}=(K / \rho)^{1 / 2}
$$

and for porous solid it can be written as

$$
C_{b}^{*}=\left(K^{*} / \rho^{*}\right)^{1 / 2},
$$

where $K^{*}$ and $\rho^{*}$ are the effective bulk modulus and effective density respectively.

To start with, $K^{*}$ is established for a single pore (sp) model with a hollow sphere approximation and then it is extended to a multipore model for which the following procedure is adopted.

Consider a hollow sphere as given in figure 1 with an outer radius $a$ and inner radius $b$. When a pressure of $P_{\mathrm{sp}}$ is applied externally, the change in outer radius $\Delta a$ is given as (Roark and Young 1975)

$$
\Delta a=-\left[P_{\mathrm{sp}} a / E_{y}\right]\left[\left\{\overline{(1-\gamma)\left(b^{3}+2 a^{3}\right)} / \overline{2\left(a^{3}-b^{3}\right)}\right\}-\gamma\right] \text {, }
$$

where $E_{y}$ is the Young's modulus and $\gamma$ the Poisson's ratio. The overall volume strain $\varepsilon_{v}$ of the hollow sphere under pressure $P_{\mathrm{sp}}$ is given as

$$
\varepsilon_{v}=3 \Delta a / a \text {. }
$$

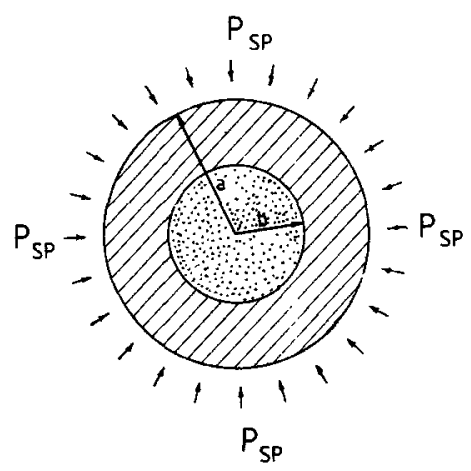

Figure 1. Single pore model (hollow sphere). 
The effective bulk modulus of this hollow sphere is

$$
K_{\mathrm{sp}}^{*}=P_{\mathrm{sp}} / \varepsilon_{v} .
$$

Porosity $\theta$ of the hollow sphere is given by

$$
\theta=b^{3} / a^{3}
$$

and the bulk modulus $K$ of the dense material is related to the Young's modulus $E_{y}$ and the Poisson's ratio $\gamma$ by

$$
K=E_{y} / 3(1-2 \gamma)
$$

Combining (8) to (12), we can obtain the effective bulk modulus $K_{\mathrm{sp}}^{*}$ in terms of $K$ and $\gamma$,

$$
K_{\mathrm{sp}}^{*}=K(1-\theta) /\left(1+b_{K} \theta\right)
$$

where

$$
b _ { K } = ( 1 + \gamma ) \longdiv { 2 ( 1 - 2 \gamma ) } .
$$

The effective bulk modulus established for single pore geometry has to be modified to make it suitable to multipore (mp) geometry. For this purpose the multipore geometry is approximated as an assembly of single pore geometries as given in figure 2. When the applied pressure is $P_{\mathrm{mp}}$ the average pressure inside the multipore solid is $P_{\mathrm{mp}} /(1-\theta)$. This has been verified using stress analysis by FEM for a multipore geometry and the finite element mesh used is given in figure 3 . Hence, the average pressure acting on single pore geometries extracted out of multipore assembly is

$$
P_{\mathrm{sp}}=P_{\mathrm{mp}} /(1-\theta) \text {. }
$$

To produce the same volume strain in the case of single pore and multipore geometries, the pressures required are different. Therefore

$$
K_{\mathrm{mp}}^{*} / K_{\mathrm{sp}}^{*}=\left\{P_{\mathrm{mp}} / \varepsilon_{v}\right\} /\left\{P_{\mathrm{sp}} / \varepsilon_{v}\right\}=1-\theta
$$

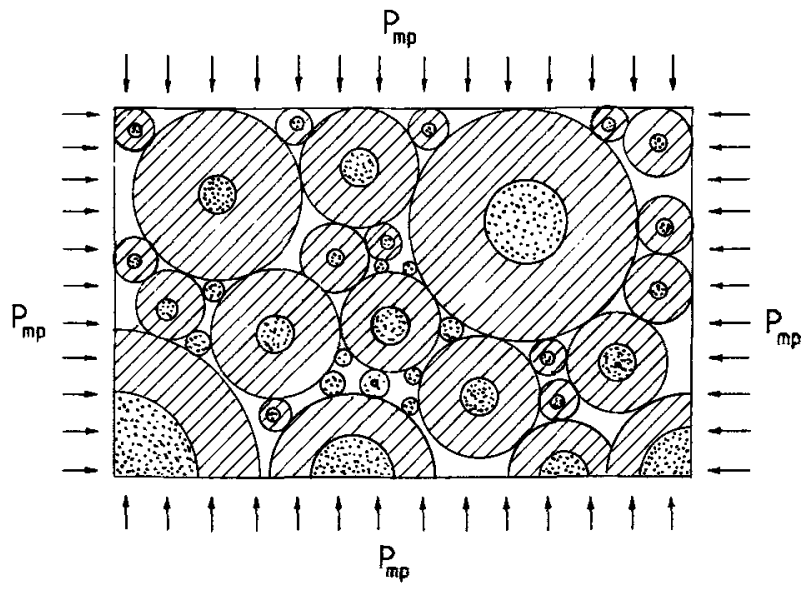

Figure 2. Multipore model (assembly of hollow spheres). 


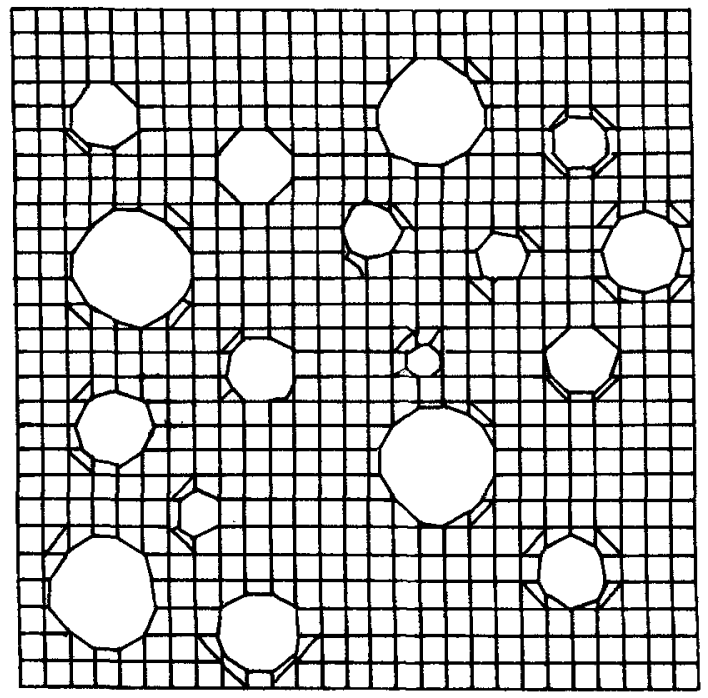

Figure 3. Finite element mesh representing multipore model.

Combining (13) and (15) we get

$$
K_{\mathrm{mp}}^{*}=K(1-\theta)^{2} /\left(1+b_{K} \theta\right) .
$$

It is to be noted here that $K^{*} / K=1$ for $\theta=0$ and vanishes for $\theta=1$, satisfying the extremities. Experimental verification of (16) is given in the Appendix. The average density of the porous solid is

$$
\rho^{*}=\rho(1-\theta) \text {. }
$$

and combining (17) with (6) and (7) we obtain

$$
C_{b}^{*}=C_{b}\left[(1-\theta) /\left(1+b_{K} \theta\right)\right]^{1 / 2} \text {. }
$$

\section{Evaluation of inverse ultimate volume strain $\boldsymbol{g}^{*}$}

The standard pressure-density relationship for a dense solid obtained by combining (1) (4) is given as

$$
P=\rho C_{b}^{2} \eta /(1-g \eta)^{2},
$$

where $\eta$ is the volume strain. For $\eta=1 / g, P \rightarrow \infty$ and hence $g$ can be understood as inverse ultimate volume strain. In this analysis, the state of material under infinite pressure is assumed to be independent of the initial state. Though this assumption breaks down for highly porous material where the internal energy increase is significantly high, for the other majority of cases it makes the analysis simpler.

Let $V_{0}$ be the specific volume of a dense solid and $V_{0}^{*}$ the specific volume of the same solid with a porosity $\theta$, where

$$
V_{0}=V_{0}^{*}(1-\theta) \text {. }
$$

Let $V_{u}$ be the specific volume corresponding to the infinite pressure. For the dense 
solid

$$
\left(V_{0}-V_{u}\right) / V_{0}=1 / g .
$$

and for the porous solids, according to the assumption,

$$
\left(V_{0}^{*}-V_{u}\right) / V_{0}^{*}=1 / g^{*} \text {. }
$$

Combining (20)-(22) we obtain

$$
g^{*}=g /\{1+\theta(g-1)\}
$$

\section{Establishing the pore collapse function}

The linear equation relating shock speed and particle speed for a dense solid as given in (4) if extended to porous solid in a similar fashion, will be of the form

$$
U_{s}^{*}=C_{b}^{*}+g^{*} U_{p} .
$$

But this equation assumes no change in the shape of the pores during shock loading. In a real situation shock loading results in pore collapse through pore wall fracture by shearing, effectively reducing the bulk sound speed. To take this into account, (24) can be modified as

$$
U_{s}^{*}=\Psi C_{b}^{*}+g^{*} U_{p}
$$

in which a pore collapse function $\Psi$ has been introduced to modulate the value of $C_{b}^{*}$. The function $\Psi$ should satisfy the following experimentally observed conditions:

(i) when $U_{p} \rightarrow 0$ for any finite $\theta, U_{s}^{*} \rightarrow 0$

(ii) when $U_{p} \rightarrow 0$ for $\theta=0, U_{s}^{*} \rightarrow$ finite value corresponding to that of dense solid. The most suitable function satisfying these conditions was found to be

$$
\Psi=\left(k_{1} U_{p} / C_{b}\right)^{k_{2}}
$$

where $k_{1}$ and $k_{2}$ are constants.

Experimental data for porous (i) 2024 aluminium, (ii) copper, (iii) iron ${ }^{1}$, (McQueen et al 1970), (iv) polyurethane foam (Butcher 1971) and (v) tungsten (Boade 1971) are plotted against the calculated values using (25) for $k_{1}=\sqrt{3}$ and $k_{2}=3 / 2$ in figure 4 and it is found to match well for different porosities, in the particle speed region of $0.25 \mathrm{~km} / \mathrm{s}$ to $5 \mathrm{~km} / \mathrm{s}$. Interestingly, $k_{1}$ and $k_{2}$ are found to be not only independent of the particle speed and porosity but also of the material.

\section{Pressure vs volume Hugoniots for loading and unloading}

During shock loading, pore collapse takes place by pore wall shearing which is not reversible during unloading. If there is no such shearing during unloading, $\Psi$ can be expressed as

$$
\Psi=\left(\sqrt{3} U_{p} / C_{b}\right)^{1 \cdot 5 \theta}
$$

for loading and

$$
\Psi=1 \text {. }
$$

\footnotetext{
${ }^{1}$ For iron $U_{s}=C_{b}+g U_{p}+q U_{p}^{2}$ and in this analysis $q$ is assumed to be independent of porosity.
} 
(a)

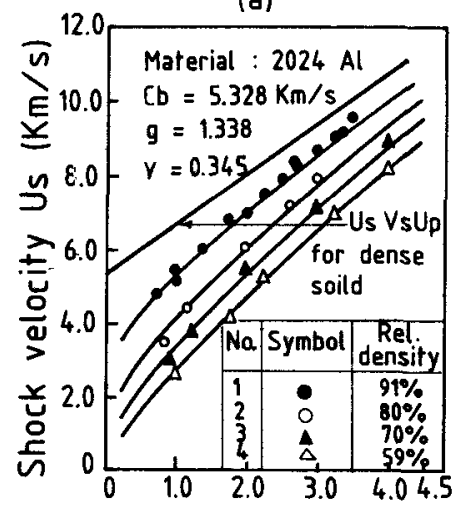

Particle velocity Up $(\mathrm{Km} / \mathrm{s})$

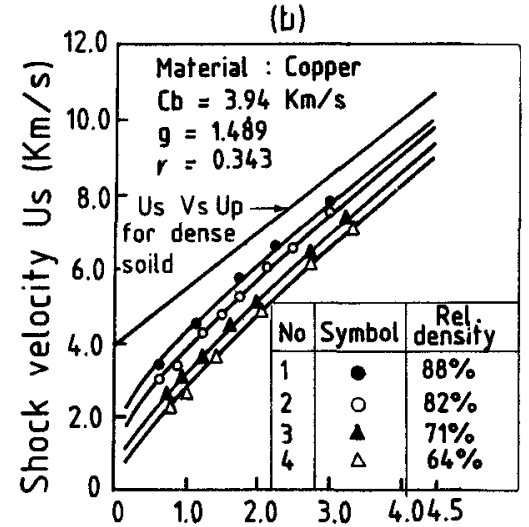

Particle velocity Up $(\mathrm{Km} / \mathrm{s})$
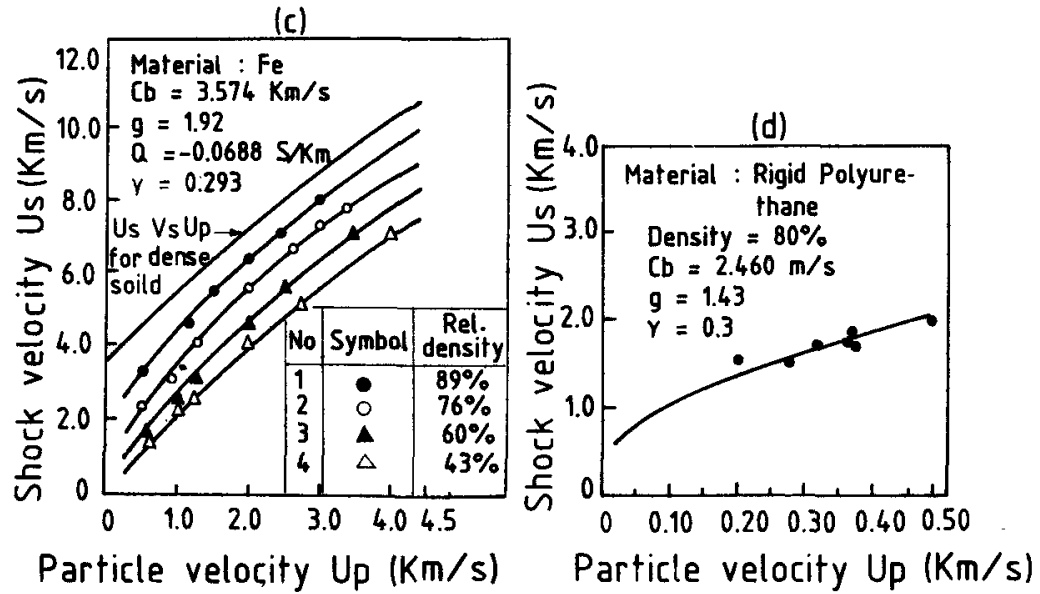

Particle velocity Up $(\mathrm{Km} / \mathrm{s})$

Particle velocity Up $(\mathrm{Km} / \mathrm{s})$

(e)

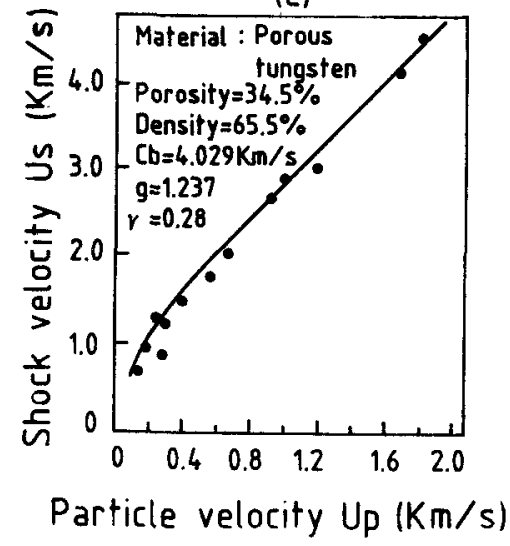

Figure 4. Particle velocity vs shock velocity. (a) 2024 aluminum, (b) copper, (c) iron. (d) polyurethane foam and (e) tungsten. 
for unloading. Combining (1), (2) and (25) with (27) and (28) independently, we get

$$
P=\rho_{0}\left(1-\theta_{0}\right)\left(\sqrt{3} / C_{b}\right)^{\left(\frac{6 \theta_{0}}{2-3 \theta_{0}}\right)}\left(\frac{C_{b}^{*}}{1-g^{*} \eta}\right)\left(\frac{4}{2-3 \theta_{0}}\right) \eta^{\left(\frac{2+3 \theta_{0}}{2-3 \theta_{0}}\right)}
$$

for loading

and $\quad \bar{P}=\frac{\rho_{0}\left(1-\theta_{f}\right) \bar{C}_{b}^{* 2} \bar{\eta}^{2}}{\left(1-\bar{g}^{*} \bar{\eta}\right)^{2}}$

for unloading, where $\rho_{0}$ corresponds to the density of the material in the dense state. Hence $\eta$ corresponds to the volume strain of the loading cycle and $\bar{\eta}$ corresponds to that of the unloading cycle and both $\eta$ and $\bar{\eta}$ are evaluated with respect to zero pressure volume of the loading and unloading cycles respectively. $C_{b}^{*}$ and $g^{*}$ correspond to $\theta_{0}$ (initial porosity) and $\bar{C}^{*}$ and $\bar{g}^{*}$ to $\theta_{f}$ (final porosity). Both $P$ and $\bar{P}$ merge with the Hugoniot of the dense solid for $\theta=0$. A typical variation of $P$ and $\bar{P}$ is shown in figure 5. If a porous material is in the state $A$ as in figure 5, for an applied impact pressure of $P$ the material reaches the state $\mathrm{B}$ and after unloading reaches $\mathrm{C}$ corresponding to the final porosity $\theta_{f}$. In figure $6 P-V$ Hugoniots corresponding to aluminum is given and these results match with the experimental results (table 1) published by Butcher et al (1974).

\section{Summary}

In the foregoing, the EOS for porous materials under shock loading has been developed based on the physical interpretation of the linear EOS of dense solids.

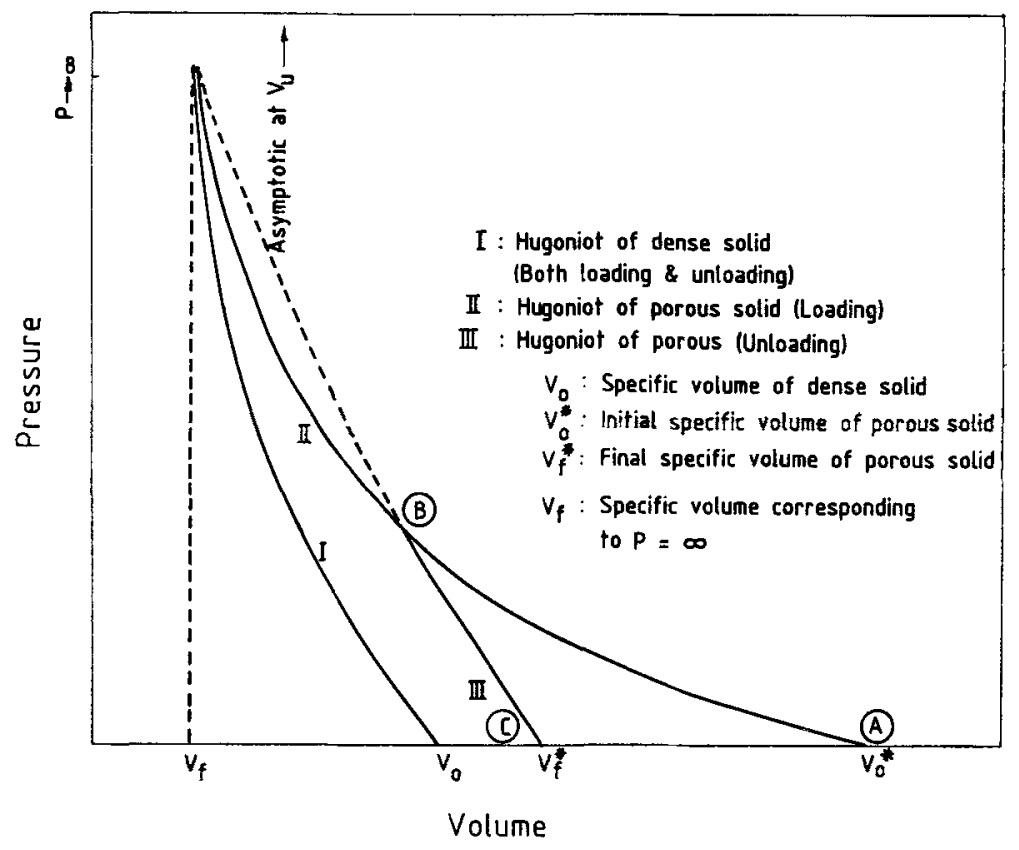

Figure 5. Hugoniot of porous solid compared to that of dense solid. 


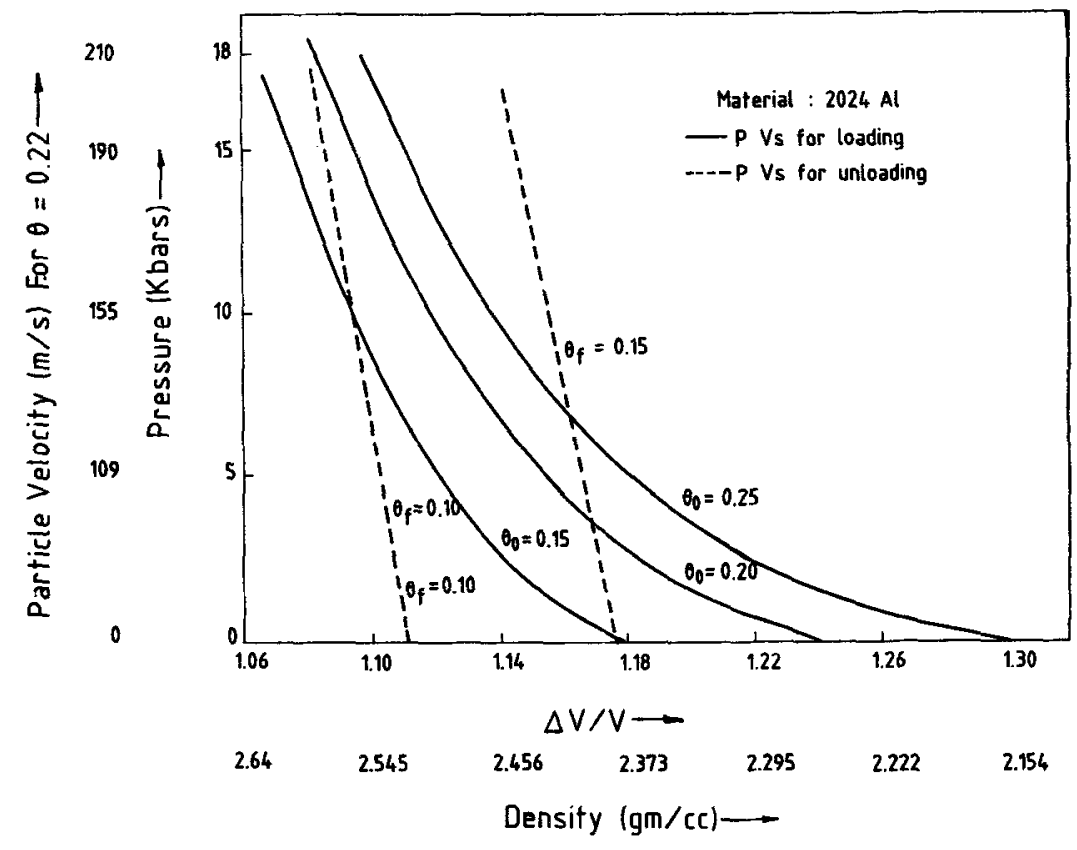

Figure 6. Hugoniot of 2024 aluminum.

Table 1. Experimental data of aluminum with initial density $2 \cdot 16 \mathrm{~g} / \mathrm{cc}$. (Butcher et al 1974).

\begin{tabular}{cc}
\hline $\begin{array}{l}\text { Particle velocity } \\
(\mathrm{m} / \mathrm{s})\end{array}$ & $\begin{array}{c}\text { Final density } \\
(\mathrm{g} / \mathrm{cc})\end{array}$ \\
\hline $57 \cdot 7$ & $2 \cdot 19$ \\
$110 \cdot 0$ & $2 \cdot 43$ \\
$112 \cdot 0$ & $2 \cdot 42$ \\
$132 \cdot 0$ & $2 \cdot 45$ \\
\hline
\end{tabular}

Effective bulk sound speed $C_{b}^{*}$, effective inverse ultimate volume strain $g^{*}$ and pore collapse function $\Psi$ are the three important parameters used to construct the EOS and its main features are as follows:

(i) This does not call for any experiments to be conducted with the porous material of interest. The material constants of the material at dense state and the initial porosity are enough to describe the equation.

(ii) The pore collapse function $\Psi$ introduced in this EOS distinguishes loading and unloading behaviour but its semi-empirical nature is a major shortcoming in this EOS. Nevertheless $\Psi$ is not only independent of porosity but also independent of the material.

\section{Appendix}

Dean and Lopez (1983) compiled the data available on the empirical dependence of 
(a)

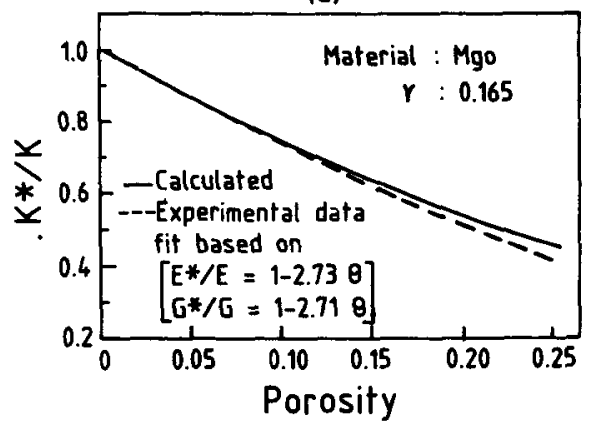

(c)

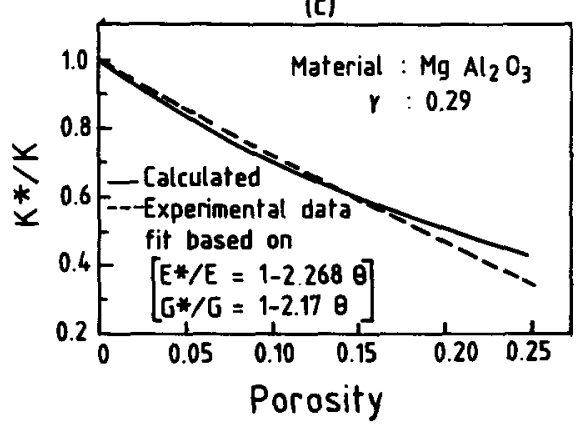

(b)

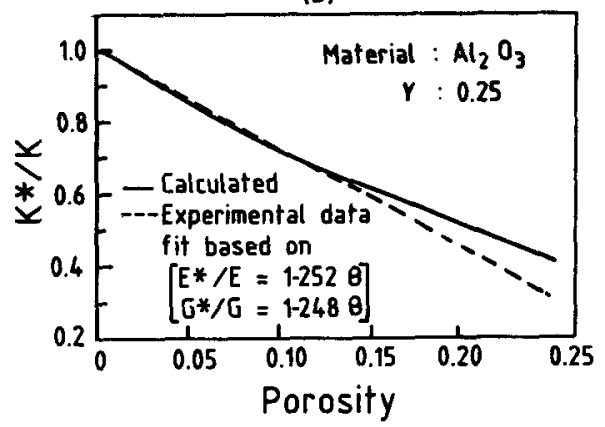

(d)

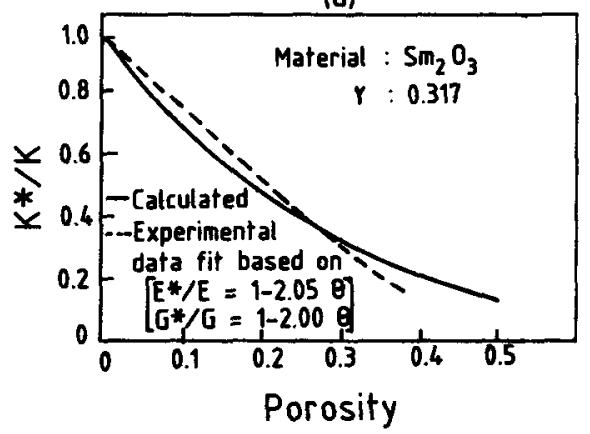

Figure 7. Experimental and calculated values of effective moduli. (a) $\mathrm{MgO}$, (b) $\mathrm{Al}_{2} \mathrm{O}_{3}$. (c) $\mathrm{Mg} \mathrm{Al}_{2} \mathrm{O}_{4}$ and (d) $\mathrm{Sm}_{2} \mathrm{O}_{3}$.

elastic moduli on porosity for ceramic materials and have presented the coefficients corresponding to the linear dependence of Young's and shear modulii on the porosity for the materials $\mathrm{Al}_{2} \mathrm{O}_{3}, \mathrm{MgO}, \mathrm{MgAl}_{2} \mathrm{O}_{3}$ and $\mathrm{Sm}_{2} \mathrm{O}_{3}$. The linear equations are given as

$$
\begin{aligned}
& E_{y}^{*} / E_{y}=1-C_{e} \theta \\
& G^{*} / G=1-C_{g} \theta
\end{aligned}
$$

Combining (31) and (32) with the standard equation

$$
K=G E_{y} / 3\left(G-E_{y}\right)
$$

we get

$$
K^{*} / K=\frac{(1-2 \gamma)\left(1-C_{g} \theta\right)\left(1-C_{e} \theta\right)}{3\left(1-C_{g} \theta\right)-2(1+\gamma)\left(1-C_{e} \theta\right)}
$$

The experimental fit obtained with (34) is compared with the calculated values of (16) in figure 7 which agrees well with the experimental data in the applicable range of porosity.

\section{References}

Al'tshuler L V, Krupnikov K K, Ledenev B N, Zhuchinkhin V I and Brazhnik M I 1958 Sov. Phys. JETP (Engl. Transl.) 7606 
Boade R R 1971 Proceedings of the 17th Sagamore Army Materials Research Conference (eds) John J Burke and Volker Weirs (Syracuse: Syracuse University Press) p. 263

Butcher B M 1971 Proceedings of the 17th Sagamore Army Materials Research Conference (eds) John J Burke and Volker Weirs (Syracuse: Syracuse University Press) p. 227

Butcher B M. Corroll M M and Holt A C 1974 J. Appl. Phys. 93864

Christensen R M 1979 Mechanics of composite materials (New York: John Wiley) p. 31

Dean E A and Lopez I A 1983 J. Am. Ceram. Soc. 66366

Derek Raybould 1981 in: Shock waves and high-strain-rate phenomena in metals (eds) Marc A Meyers and Lawrence A Murr (New York: Plenum Press) p. 895

Hermann W 1969 J. Appl. Phys. 402490

Linde R K and Schmidt D N 1966 J. Appl. Phys. 373259

Lotrich V F, Tamotsu Akashi and Akira Sawaoka 1986 in: Metallurgical applications of shock-wave and high-strain-rate phenomena (eds) Lawrence E Murr, Karl P Standhammer and Marc A Meyers (New York: Marcel Dekker Inc.) p. 227

McQueen R G, Marsh S P, Taylor J W, Fritz J N and Carter W J 1970 in: High velocity impact phenomena (ed.) Ray Kinslow (New York: Academic Press) p. 294

Roark R J and Young W C 1975 Formulae for stress and strain (Tokyo: McGraw-Hill Kogakusha Ltd) (fifth edition) p. 506

Roman O V and Gorobtsov V G 1981 in: Shock waves and high-strain-rate phenomena in metals (eds) Marc A Meyers and Lawrence E Murr (New York: Plenum Press) 827

Simons A and Legner H H 1982 J. Appl. Phys. 53943

Zel'Dovich Y and Raizer Y 1967 in: Physics of shock waves in high-temperature hydrodynamic phenomena (eds) Wallace D Hayes and Ronald F Probstein (New York: Academic Press) Vol. 2 p. 712 